\title{
Active control of mechanical distributed systems with stochastic parametric excitations
}

\author{
Andrzej Tylikowski* \\ Warsaw University of Technology \\ Institute of Machine Design Fundamentals, Narbutta 84, \\ 02-524 Warsaw, Poland. Phone: 0482-6608244. Fax: 04822-490306. \\ $E$-mail: aty@syriusz.simr.pw.edu.pl
}

\begin{abstract}
The study is based on the application of piezoelectric distributed sensors, actuators, and an appropriate feedback and is adopted for stability problems of system consisting of plate with control part governed by partial differential equations with stochastic coefficients. The application of Liapunov method to the Itô equation leads to the effective analytical estimation of a gain velocity feedback implying nonincreasing of the functional along an arbitrary plate motion and in consequence to balance the supplied energy by the parametric excitation and the dissipated energy by the inner and control damping.

Keywords

stochastic systems, distributed control, Liapunov method, stabilization, elastic plates, piezoelectric transducers
\end{abstract}

\section{INTRODUCTION}

Piezoelectric materials show great advantages as sensors and actuators in intelligent structures i.e. structures with highly distributed actuators, sensors, and processor networks. Piezoelectric sensors and actuators have been applied successfully in the closed-loop control (cf. Bailey and Hubbard, 1985). Crawley and de Luis (1987) presented a comprehensive static model for a piezoelectric actuator glued to a beam. This one-dimensional theory was extended by Dimitriadis, Fuller and Rogers (1991) on thin plates with twodimensional piezoelectric patches. The general dynamic coupling model of the beam with bonding sensors and actuators was used to derive the Liapunov control strategy, which

*This research was supported by the grant from the Polish State Committee for Scientific Research (KBN Nr 3P4 04009 07) 
is especially useful in the collocated sensor-actuator systems (Tylikowski, 1993). The dynamic extensional strain on the beam surface was calculated by considering the dynamic coupling between the actuator and the beam, and by taking into account a finite bonding layer with the finite stiffness (Tylikowski, 1994). Lee and Moon (1990) introduced novel distributed sensors/actuators, which sense and actuate the particular modal coordinate without extensive signal processing. Dimitriades, Fuller and Rogers (1991) showed that two dimensional patch type actuators show large potential for controlling vibration in distributed systems. Lee, Chiang and O'Sulivan (1991) demonstrated advantages of combining distributed modal sensor/actuator pairs into flexible structures. Tzou and Fu (1992) analysed models of a plate with segmented distributed piezoelectric sensors and actuators, and showed that segmenting improves the observability and the controllability of the system. Recently designed by Kumar, Bhalla, and Cross (1994) the piezoelectric actuators with constant properties over a wide-band frequencies seems to be appropriate for applying in systems subjected to wide-band stochastic excitations. Chow and Maestrello (1993) examined the exponential stabilization of panels with time-dependent parametric excitations and concluded that a stronger mode of control, such as a distributed control, should be used to stabilize time-dependent systems. The direct Liapunov method was applied to the stabilization problem of the beam subjected to a wide-band parametric excitation (Tylikowski, 1995).

The purpose of the present paper is to solve an active control problem of parametric vibrations excited by the biaxial in-plane wide-band Gaussian forces. The problem is solved using the concept of distributed piezoelectric sensors and actuators with a sufficiently large value of velocity feedback. Real mechanical systems are subjected not only to nontrivial initial conditions but also to permanently acting excitations and the active vibration control should be modify in order to balance the supplied energy by external parametric excitation. The applicability of active vibration control is extended to distributed systems with stochastic parametric excitation. The effective estimation of the feedback constant stabilizing the plate parametric vibration is derived analytically. The minimal value of the feedback constant is effectively expressed by the constant component of in-plane forces, intensities of stochastic components, geometry, mechanical and piezoelectric properties of actuators and sensors.

\section{DYNAMICS EQUATION}

Consider a Kirchhoff plate of a bending stiffness $D$ biaxially loaded in the plate middle plane by time-dependent forces $F_{x}$, and $F_{y}$ uniformly distributed over the simply supported edges. The rectangular plate domain is denoted by $\mathcal{P} \equiv(0, a) \times(0, b)$. The dynamics equation of plate motion includes both an internal passive damping with the proportionality coefficient $\alpha$ and an active damping. Thin piezoelectric patches are perfectly mounted on opposite sides of the plate. It is assumed that the transverse motion dominates the in-plane plate vibrations. The thickness of the plate, the actuator and the sensor is denoted by $t_{p}, t_{a}$ and $t_{s}$, respectively. The sensing and actuating effects of piezoelectric layers are used to extract the mechanical energy and in a final result to stabilize both the free vibration due to initial disturbances and the parametric vibration excited by the axial force. Assume a negligible stiffness of the sensor in comparison with that of 
the plate and reducing the influence of the piezoelectric actuator on the plate to bending moments $M_{x}, M_{y}$ distributed over the plate surface.

\subsection{Sensor equation}

Sensor electric displacement

$D_{3}=-e_{s_{31}} \epsilon_{s_{1}}-e_{s_{32}} \epsilon_{s_{2}}$

Substituting Hook's law and the geometric formulas relating deformations on the plate surface with the curvatures of the middle plane $\epsilon_{s_{1}}=\left(t_{p}+t_{s}\right) w_{, x x} / 2, \epsilon_{s_{2}}=\left(t_{p}+t_{s}\right) w_{, y y} / 2$ we have

$D_{3}=-\frac{\left.t_{p}+t_{s}\right) E_{s}}{2\left(1-\nu_{s}^{2}\right)}\left[\left(d_{s_{31}}+\nu d_{s_{32}}\right) w_{, x x}+\left(d_{s_{32}}+\nu d_{s_{31}}\right) w_{, y y}\right]$

Integrating the electric displacement over the sensor area $\mathcal{S}$ and dividing by the capacity we obtain the sensor voltage of the open circuit

$\mathcal{V}_{s}=-\frac{t_{s}\left(t_{p}+t_{s}\right) E_{s}}{2 A_{s} \varepsilon_{33}\left(1-\nu_{s}^{2}\right)} \int_{\mathcal{S}}\left[\left(d_{s_{31}}+\nu_{s} d_{s_{32}}\right) w_{, x x}+\left(d_{s_{32}}+\nu_{s} d_{s_{31}}\right) w_{, y y}\right] d \mathcal{S}$

\subsection{Actuator equation}

Normal stresses in the actuator due to the direct piezoelectric effect are as follows

$\sigma_{a_{1}}=\frac{d_{a_{31}} \mathcal{V}_{a}}{t_{a}}$

$\sigma_{a_{2}}=\frac{d_{a_{32}} \mathcal{V}_{a}}{t_{a}}$

Assuming the uniform stress distribution over the actuator thickness the control bending moments are given by the following formulas

$\left[\begin{array}{l}M_{x} \\ M_{y}\end{array}\right]=\frac{E_{a} \mathcal{V}_{a}\left(t_{p}+t_{a}\right)}{2\left(1-\nu_{s}^{2}\right) t_{a}} \chi_{a}(x, y)\left[\begin{array}{c}\left.d_{a_{31}}+\nu_{a} d_{a_{32}}\right) w_{, x x} \\ \left(d_{a_{32}}+\nu_{a} d_{a_{31}}\right) w_{, y y}\end{array}\right]$

The shape of the piezoelectric actuator $\mathcal{A}$ is described by the characteristic function $\chi_{a}(x, y)$.

\subsection{Plate equation of motion}

Assuming the velocity feedback control with the gain factor $K_{a}$ the voltage applied to the actuator is given by

$\mathcal{V}_{a}=K_{a} \frac{d \mathcal{V}_{s}}{d t}$ 
The plate differential equation of motion with closed loop control has the form

$\rho t_{p} w_{, t t}+\alpha w_{, t}+F_{x}(t) w_{, x x}+F_{y}(t) w_{, y y}+D \Delta^{2} w+M_{x, x x}+M_{y, y y}=0, \quad(x, y) \in \mathcal{P}$.

Substituting the bending moments and introducing dimensionless coordinates yield

$$
\begin{aligned}
& w_{, t t}+2 \beta w_{, t}+\left(f_{o x}+f_{x}(t)\right) w_{, x x}+\left(f_{o y}+f_{y}(t)\right) w_{, y y}+\Delta^{2} w+ \\
& +2\left(\beta_{x} \chi_{a, x x}+\beta_{y} \chi_{a, y y}\right) S\left(w_{, t}\right)=0
\end{aligned}
$$

where the sensor linear functional has the form

$$
S(w)=\int_{\mathcal{S}}\left(\left(d_{31}+\nu d_{32}\right) w_{, x x}+\left(d_{32}+\nu d_{, 31}\right) w_{, y y}\right) d \mathcal{S} .
$$

The actuator shape is described by the characteristic function $\chi_{a}(x, y)$. Equation (9) with zero initial conditions $w(x, 0)=\frac{\partial w}{\partial t}(x, 0)=0$ possess a trivial solution $w(x, t)=0$, which corresponds to an undeflected plate middle plane.

From the mathematical point of view, the feature common to all parametric vibrations is that they are described by differential equations with coefficients depending explicitly on time. In deterministic parametric vibrations it is well known that the stability properties are determined from the Mathieu equation together with the corresponding Ince-Strutt diagram. If the parametric excitation becomes random, the stability criteria depend on the statistical characteristics of the excitation and the system parameters. Specifically, if the excitation is sufficiently narrow-banded or it has one latent periodicity, a series of wedges on the amplitude-frequency plane can be expected, analogously to the deterministic parametric resonance. In the present analysis the direct Liapunov method is proposed so as to solve stochastic parametric vibration and to establish a stability criterion for the equilibrium state of plate with closed-loop control in a stochastic sense.

\section{ENERGY EXTRACTION}

Vibration damping of the plate with parametric excitation can be examined by means of the total energy considerations. The mechanical energy consists of the kinetic energy, the bending energy, and the elastic energy of compression due to the constant components of in-plane forces $f_{o x}$, and $f_{o y}$

$$
V=\frac{1}{2} \int_{\mathcal{P}}\left(w_{, t}^{2}+(\Delta w)^{2}-f_{o x} w_{, x}^{2}-f_{o y} w_{, y}^{2}\right) d \mathcal{P}
$$

The energy is positive-definite if the classic buckling condition is fulfilled by the constant components of in-plane forces. By differentiating equation (11) with respect to time, the rate of energy extraction is

$$
\frac{d V}{d t}=\int_{\mathcal{P}}\left(w_{, t} w_{, t t}+\Delta w \Delta w_{, t}-f_{o x} w_{, x} w_{, x t}-f_{o y} w_{, y} w_{, y t}\right) d \mathcal{P}
$$


Eliminating the acceleration by means of dynamics equation (9), and integrating by parts (12) gives

$$
\begin{aligned}
& \frac{d V}{d t}=-\int_{\mathcal{P}} w_{, t}\left(f_{x}(t) w_{, x x}+f_{y}(t) w_{, y y}\right) d \mathcal{P}-2 \beta \int_{\mathcal{P}} w_{, t}^{2} d \mathcal{P}+ \\
& -2 S\left(w_{, t}\right) \int_{\mathcal{A}}\left(\beta_{x} \chi_{a, x x}+\beta_{y} \chi_{a, y y}\right) w_{, t} d \mathcal{P}
\end{aligned}
$$

The first component with an undefined sign in equation (13) is the power flow due to the parametric excitation. The second negative component represents the rate at which the energy is extracted from the plate by the passive damping. Assuming the same shape of sensor and actuator the third component in equation (13) also describes the energy extraction as it can be expressed by the square of integral. Therefore, for the sufficiently large gain factor it is possible to stabilize parametric vibrations excited by the time-dependent axial force.

The analogous analyses of active damping vibration caused due to initial nonzero disturbances were presented by a number of researchers (eg. Bailey, and Hubbard, (1985), and recently Pourki, (1993)). However, inequality (13) does not provide an effective quantitive estimation of the minimal active damping coefficients $\beta_{x}, \beta_{y}$ stabilizing the parametric vibration. In order to derive an analytical relation involving characteristics of the parametric excitation, and parameters of passive and active damping it is necessary to precisely define the class of parametric excitations $f_{x}(t), f_{y}(t)$ randomly fluctuating over a wide-band of frequencies and reformulate the stabilization problem of plate parametric vibration as a qualitative analysis of stochastic partial differential equations (Curtain, and Falb, 1965). More advanced methods of stability analysis introduced for distributed parameter systems with wide-band Gaussian coefficients (Tylikowski, 1991) have to be applied to derive the formula describing the stability domains as a function of the passive and active damping coefficients, and statistical characteristics of parametric excitation.

\section{STOCHASTIC STABILITY PROBLEM}

If the parametric excitation is a wide-band Gaussian stochastic process equation (9) should be understood as the Itô stochastic partial differential equation (Curtain, and Falb, 1965), and rewritten in the form

$$
\begin{aligned}
& d w=w_{, t} d t \\
& d w_{, t}=-\left(2 \beta w_{, t}+\Delta^{2} w+f_{o x} w_{, x x}+f_{o y} w_{, y y}+2\left(\beta_{x} \chi_{A_{, x x}}+\beta_{y} \chi_{A_{, y y}}\right) S(w)\right) d t+ \\
& -\sigma_{x} w_{, x x} d \mathcal{W}_{x}-\sigma_{y} w_{, y y} d \mathcal{W}_{y}, \quad(x, y) \in \mathcal{P} .
\end{aligned}
$$

where $\mathcal{W}_{x}, \mathcal{W}_{y}$ are independent Wiener processes, and $\sigma_{x} \sigma_{y}$ the intensities of the compressive forces equal to the square roots of their maximal spectral densities. As there is no randomness in the first equation we notice that the Stratonovich-Wong-Zakai correction term is equal to zero.

We assume that the solutions for equations (14) and (15) exist and belong to the 
appropriate Hilbert space. The purpose of the present paper is to derive criteria for solving the following problem: will the deviations of the plate middle plane from the unperturbed state (trivial solution) be sufficiently small in some mathematical sense in the case when the in-plane forces are the wide-band Gaussian processes. The plate dynamically buckles when the in-plane forces get so large that the plate with closed-loop control does not oscillate and a new increasing mode of oscillations occurs. To estimate a perturbed solution of equations (14) and (15) it is necessary to introduce a measure of distance $\|$.$\| of the$ solution of equations (14) and (15) with nontrivial initial conditions from the trivial one. The equilibrium state of equation (14) and (15) is said to be uniformly stochastically stable, if the following logic sentence is true

$$
\bigwedge_{\epsilon \geq 0} \bigwedge_{\delta \geq 0} \bigvee_{r \geq 0}\|w(., 0)\| \leq r \Rightarrow P\left(\sup _{t \geq 0}\|w(., t)\| \geq \epsilon\right) \leq \delta
$$

In the present paper the direct Liapunov method is proposed to establish criteria for the uniform stochastic stability of the unperturbed (trivial) solution of the plate with closed-loop control.

\section{STABILITY ANALYSIS}

We construct the Liapunov functional as a sum of the modified kinetic energy and the elastic energy of the plate (Tylikowski, 1991)

$$
V=\frac{1}{2} \int_{\mathcal{P}}\left[\left(\Delta^{2} w\right)^{2}-f_{o x} w_{, x x}^{2}-f_{o y} w_{, y y}^{2}+\left(w_{, t}+2 \beta w+2\left(\beta_{x} \chi_{a, x x}+\beta_{y} \chi_{a, y y}\right) S(w)\right)^{2}\right] d \mathcal{P} .(17)
$$

If the classical condition for the static buckling is fulfilled, functional (17) satisfies the positive-definiteness condition, and the measure of distance between the perturbed solution and the trivial one can be chosen as the square root of the functional $\|\cdot\|=V^{1 / 2}$. As realizations of the Wiener process are not differentiable the Itô calculus has to be applied to calculate the differential of functional (17)

$d V=\mathcal{F}(w) d t+\mathcal{G}_{x} d \mathcal{W}_{x}+\mathcal{G}_{x} d \mathcal{W}_{y}$

where

$$
\begin{aligned}
& \mathcal{F}=\int_{\mathcal{P}}\left[2\left(\beta+\left(\beta_{x} \chi_{a, x x}+\beta_{y} \chi_{a, y y} S(w)\right)\right)(\Delta w)^{2}-\frac{1}{2}\left(\sigma_{x}^{2} w_{, x x}^{2}+\sigma_{y}^{2} w_{, y y}^{2}\right)+\right. \\
& \left.-2 \beta\left(f_{o x} w_{, x}^{2}+f_{o y} w_{, y}^{2}\right)-2 S(w)\left(\beta_{x} \chi_{a, x x}+\beta_{y} \chi_{a, y y}\right)\left(f_{o x} w_{, x x}+f_{o y} w_{, y y}\right)\right] d \mathcal{P} .
\end{aligned}
$$

Integrating the differential (18) with respect to time from $s$ to $\tau_{\delta}(t)$, where $\tau_{\delta}(t)=$ $\min \left(t, \tau_{\delta}\right)$, and $\tau_{\delta}$ is a random time of the first exit from the domain $\|\cdot\|=\delta$, conditionally averaging $(\mathbf{E})$ with respect to a $\sigma$-algebra $\mathcal{N}_{t} \subset B$ generated by the Wiener processes 
$\left\{\xi\left(s^{\prime}\right) \mid s^{\prime} \subset[s, t]\right\}$ and taking into account the fact that the conditional average of the second and third terms in equation (18) are equal to zero, we have

$\mathbf{E} V\left(\tau_{\delta}(t)\right)=V(s)-\mathbf{E} \int_{s}^{\tau_{\delta}(t)} \mathcal{F} d t$

Assuming that

$\mathcal{F} \geq 0$

we can write the following inequality

$\mathbf{E} V\left(\tau_{\delta}(t)\right) \leq V(s)$

Proceeding similarly to the proof of Chebyshev's inequality, we find that the trivial solution (corresponding to the equilibrium state) of the stochastic equations (14) and (15) governing the dynamics of the plate with closed-loop control is uniformly stochastically stable with respect to the measure $\|\cdot\|=V^{1 / 2}$, if the inequality (21) holds.

\section{STABILIZATION CONDITIONS}

Using the derived formula (21) it is easy to obtain particular results relating to basic problems of active and passive damping of vibration. Neglecting the constant components of in-plane forces and the passive damping, and introducing the ratio of piezoelectric properties $r=\left(d_{31}+\nu d_{32}\right) /\left(d_{32}+\nu d_{31}\right)$ the minimum active damping coefficient stabilizing the parametric vibration due to wide-band Gaussian force with the known maximum spectral densities is defined in the following way

$\frac{\sigma_{x}^{2}}{4 \beta_{x}} \leq \min _{m, n=1,2, . .} \frac{\int_{\mathcal{A}} \Delta^{2}\left(w_{, x x}+\frac{\beta_{y}}{\beta_{x}} w_{, y y}\right) d \mathcal{A} \int_{\mathcal{S}}\left(w_{, x x}+r w_{, y y}\right) d \mathcal{S}}{\int_{\mathcal{P}}\left(w_{, x x}^{2}+\left(\frac{\sigma_{y}}{\sigma_{x}}\right)^{2} w_{, y y}^{2}\right) d \mathcal{P}}$.

We look for the minimum of functional in the class of functions satisfying simply supported boundary conditions $\sin (n \pi x) \sin (n \pi x)$ for $m, n=1,2, \ldots$. For the same shape of piezoelectric sensor and actuator described by Pourki's function $y=x^{2}(x-1)(1993)$, and assuming the same properties of sensor and actuator $(\mathrm{r}=1)$, as well as $\sigma_{y} / \sigma_{x}=1$ the stabilization conditions of the square plate $(a=b=1)$ has the form

$\frac{\sigma_{x}^{2}}{4 \beta_{x}} \leq 0.0135$

\section{CONCLUSIONS}

By means of the direct Liapunov method the active stabilization of a vibrating plate with distributed piezoelectric sensor, actuator, and the velocity feedback has been studied. The elastic plate is simply supported and loaded biaxially by in-plane compressive forces 
randomly fluctuating over a wide band of frequencies. Without any passive damping and control, the plate motion is unstable due to the parametric excitation.

The stabilization of stochastic parametric vibrations needs sufficiently large active damping coefficient proportional to the gain factor. The minimum gain factor stabilizing the system depends on the shape of sensor and actuator.

For no in-plane compression, this is the case of free vibration due to the nontrivial initial conditions. As long as the active or passive damping is present, the system is stable and oscillations decay.

\section{REFERENCES}

Bailey, T, and Hubbard, J. E. Jr, (1985) Distributed piezoelectric-polymer active vibration control of a cantilever beam, J. Guidance, Control, and Dynamics, 8, 605-11.

Chow, P. L, and Maestrello (1993), Stabilization of the nonlinear vibration of an elastic panel by boundary control, [in:] R. A. Burdiso, (ed.) Second Conference on Recent Advances in Active Control of Sound and Vibration, Technomic Publishing, LancasterBasel, 660-7.

Crawley, E. F, and de Luis, J, (1987) Use of piezoelectric actuators as elements of intelligent structures, it AIAA J, 25, 1373-85.

Curtain, R. F, and Falb, P. L, (1965) Stochastic differential equations in Hilbert space, J. Diff. Equations, 10, 412-30.

Dimitriadis, E, Fuller, C. E, and Rogers, C. A, (1991) Piezoelectric actuators for distributed vibration excitation of thin plates, J. Appl. Mech., 113, 100-7.

Kumar, S, Bhalla, A. S, and Cross, L. E, (1994) Smart ceramics for broadband vibration control J. Intelligent Material Systems and Structures, 5, 673-7.

Lee, C. K, Chiang, W. W, and O'Sulivan, T. C, (1991) Piezoelectric modal sensor/actuator pairs for critical active damping vibration control, J. Acoust. Soc. Am., 90, 374-84.

Pourki, F, (1993), Active distributed damping of flexible structures using piezoelectric actuator/sensors, 20, 279-85.

Tylikowski, A, (1991) Stochastic stability of continuous systems, Polish Scientific Publishers, Warszawa, (in Polish).

Tylikowski, A, (1993) Stabilization of beam parametric vibrations, Journal of Theoretical and Applied Mechanics, 32, 657-70.

Tylikowski, A, (1995) Active stabilization of beam vibrations parametrically excited by wide-band Gaussian force, [in:] S. D. Sommerfelt, and H. Hamada (eds.), Proceedings of The International Symposium on Active Control of Sound and Vibration, Newport Beach, CA, 91-102.

Tzou, H. S, and Fu, H. Q, (1992) A study on segmentation of distributed piezoelectric sensors and actuators; Part 1 - Theoretical analysis, Active Control of Noise and Vibration, ASME, DSC - 38, 239-46. 